\section{Metabolic improvement by telmisartan beyond angiotensin receptor blockade: role of adipokines}

\author{
Melhora da função metabólica ocasionada pelo telmisartan além \\ do bloqueio do receptor da angiotensina: papel das adipocinas
}

Amirhossein Sahebkar

$\mathrm{R}$ ecently, there have been promising findings on the protective effects of telmisartan against insulin resistance and related complications. Notably, amelioration of diet-induced obesity, insulin resistance, and fatty liver caused by telmisartan treatment have recently been reported in ATla-deficient mice, implying that these effects could be exerted independent of angiotensin II type 1 receptor (ATl) blockade (1). As for the mechanisms behind observed metabolic improvements, adipose tissue has been suggested as a potential target for the insulin-sensitizing effect of telmisartan. Based on this, I would like to propose an important mechanism by which adipose tissue might be involved in the observed ATl-independent effects of telmisartan.

Over the past decade, the attitude toward adipose tissue has changed from a sole energy storage organ to an active endocrine organ capable of expressing and secreting different adipokines with important metabolic activities (2). Adiponectin is an adipocyte-derived hormone that possesses interesting beneficial impacts on different metabolic processes. Numerous studies have reported the anti-obesity actions of adiponectin, as well as the improvement of multiple obesity-associated metabolic disorders, such as insulin resistance, type 2 diabetes, nonalcoholic fatty liver disease, dyslipidemia, and cardiovascular disease $(2,3)$. Hypoadiponectinemia has been proposed as a risk factor for a number of those metabolic disorders (3). Particularly, telmisartan has been found to clinically increase plasma concentrations of adiponectin, high molecular weight (HMW) adiponectin - which is the major bioactive isoform of the hormone - and $\mathrm{HMW}$-adiponectin/total adiponectin ratio (4-6). Besides, there is evidence indicating that telmisartan treatment stimulates adiponectin transcription in adipocytes $(7,8)$.

Tumor necrosis factor- $\alpha$ (TNF- $\alpha$ ) represents another important adipokine that has been shown to be critically involved in the development of insulin resistance, diabetes mellitus, lipid metabolism, nonalcoholic fatty liver disease, and obesity. Adipocyte expression and circulating levels of this cytokine have been reported to be elevated in obese or diabetic subjects. In a previous clinical study in patients with type 2 diabetes and metabolic syndrome, treatment with telmisartan was reported to significantly reduce serum levels of TNF- $\alpha(9)$. Although the impact of telmisartan on adipocyte-derived TNF- $\alpha$ needs to be clarified, it appears that this drug could change the TNF- $\alpha$ / adiponectin balance in favor of adiponectin.

Telmisartan has also been shown to reduce serum levels of resistin in obese mice, as well as in type 2 diabetic patients with metabolic syndrome $(10,11)$. Resistin is another adipokine hypothesized to link obesity, insulin resistance and type 2 diabetes. Finally, telmisartan has been demonstrated to stimulate the release of visfatin from adipocytes (12). Considering the physiological effects of visfatin, its boosted release may result in insulin-sensitizing, antidiabetic and cardioprotective effects.
Cardiovascular Research Center, Avicenna Research Institute, Mashhad University of Medical Sciences (MUMS), Mashhad, Iran. Biotechnology Research Center and School of Pharmacy, MUMS, Mashhad, Iran I

Correspondence to:

Amirhossein Sahebka sahebkarah811@mums.ac.ir

Received on Feb/10/2011 Accepted on Jun/3/2011 
Taken together, ATl-independent, beneficial metabolic effects of telmisartan against obesity, insulin resistance and fatty liver may be, at least in part, attributed to the alteration of adipokine levels (including adiponectin, resistin and TNF- $\alpha$ ) produced by this drug.

Disclosure: no potential conflict of interest relevant to this article was reported.

\section{REFERENCES}

1. Rong X, LiY, Ebihara K, Zhao M, Naowaboot J, Kusakabe T, et al. Angiotensin II type 1 receptor-independent beneficial effects of telmisartan on dietary-induced obesity, insulin resistance and fatty liver in mice. Diabetologia. 2010;53:1727-31.

2. Fasshauer M, Paschke R. Regulation of adipocytokines and insulin resistance. Diabetologia. 2003;46:1594-603.

3. Sowers JR. Endocrine functions of adipose tissue: focus on adiponectin. Clin Cornerstone. 2008;9:32-8.

4. Makita S, Abiko A, Naganuma Y, MoriaiY, Nakamura M. Potential effects of angiotensin II receptor blockers on glucose tolerance and adiponectin levels in hypertensive patients. Cardiovasc Drugs Ther. 2007;21:317-8.

5. Makita S, Abiko A, Naganuma Y, Moriai Y, Nakamura M. Effects of telmisartan on adiponectin levels and body weight in hypertensive patients with glucose intolerance. Metabolism. 2008;57:1473-8.
6. Satoh M, Tabuchi T, Minami Y, Takahashi Y, Itoh T, Nakamura M. Prospective, randomized, single-blind comparison of effects of 6 months of treatment with telmisartan versus enalapril on high-molecular-weight adiponectin concentrations in patients with coronary artery disease. Clin Ther. 2009;31:2113-25.

7. Moriuchi A, Yamasaki $H$, Shimamura M, Kita A, Kuwahara $H$, Fujishima $K$, et al. Induction of human adiponectin gene transcription by telmisartan, angiotensin receptor blocker, independently on PPAR- $\gamma$ activation. Biochem Biophys Res Commun. 2007;356:1024-30.

8. Yamada S, Ano N, Toda K, Kitaoka A, Shiono K, Inoue G, et al. Telmisartan but not candesartan affects adiponectin expression in vivo and in vitro. Hypertens Res. 2008;31:601-6.

9. Derosa G, Cicero AFG, D'Angelo A, Ragonesi PD, Ciccarelli L, Piccinni MN, et al. Telmisartan and irbesartan therapy in type 2 diabetic patients treated with rosiglitazone: Effects on insulin-resistance, leptin and tumor necrosis factor- $\alpha$. Hypertens Res. 2006;29:849-56.

10. Derosa G, Fogari E, D'Angelo A, Cicero AF, Salvadeo SA, Ragonesi $P D$, et al. Metabolic effects of telmisartan and irbesartan in type 2 diabetic patients with metabolic syndrome treated with rosiglitazone. J Clin Pharm Ther. 2007;32:261-8.

11. Araki K, Masaki T, Katsuragi I, Tanaka K, Kakuma T, Yoshimatsu H. Telmisartan prevents obesity and increases the expression of uncoupling protein 1 in diet-induced obese mice. Hypertension. 2006;48:51-7.

12. Storka A, Vojtassakova E, Mueller M, Kapiotis S, Haider DG, Jungbauer $A$, et al. Angiotensin inhibition stimulates PPAR $\gamma$ and the release of visfatin. Eur J Clin Invest. 2008;38:820-6. 